\title{
Review of: "Assessment of Hydration Process And Mechanical Properties of Cemented Paste Backfill By Rebound Method"
}

Xuepeng Song

Potential competing interests: The author(s) declared that no potential competing interests exist.

In this paper, the rebound method in the field of concrete is introduced and improved, and the functional relationship between CPB hardness and UCS is established. It is considered that the rebound method can be used as a nondestructive testing method to simply and quickly predict the UCS of CPB. The article is innovative, but the following modifications still need to be made.

(1) The title of the article is "assessment of hydration process and mechanical properties of cemented paste backfill by rebound method", however, the hydration process is not reflected in the summary. In addition, after reading the full text, I found that the whole article only mentioned the internal and external hardness changes caused by the gap between the internal and external cement hydration reaction of CPB sample, and did not continue to quantify the hydration process. Therefore, I think this article mainly studies the relationship between hardness and strength, and the title of the article may need to be modified.

(2) The author says the batching and filling of concrete are the same, which are composed of aggregate, cement and water. The difference between the two only lies in the diverse types of aggregate.

(3) Generally, the last part of the introduction needs to introduce the test steps of this paper and give the research significance.

(4) The author should describe the particle size distribution of tailings, such as $D_{10}, D_{50}, D_{60}, D_{90}$, nonuniformity coefficient and curvature coefficient of tailings particles.

(5) Please explain why the middle section position is selected for rebound test.

(6) The author says the better the cementation of the test specimens, the greater the internal friction angle is. Please use relevant literature to prove your correctness. In addition, some explanations of the author also need to be proved by references. 
(7) In this paper, the strength of the center and outer surface of the sample are compared, and the relationship between the local strength and the surface hardness of the sample is obtained. I am more interested in the method of obtaining the center strength, outer surface strength and local strength. Please introduce. In addition, the principle of rebound method should be supplemented in this paper.

(8) Conclusion 5 can not be used as a conclusion, but can be used as the research significance of the article. 